\title{
Sensor validation for wearable monitoring system in ambulatory monitoring: application to textile electrodes
}

\author{
G. Andreoni, A. Fanelli, I. Witkowska, P. Perego, M. Fusca, M. Mazzola, M. G. Signorini
}

\begin{abstract}
This paper discusses the reliability of textile sensors in wearable monitoring application. A methodology for the validation of new textile electrodes for monitoring subjects in daily activities is proposed. The protocol is divided in 2 phases: a laboratory set of tests to verify the "absolute" reliability of the electrodes; a second set of trials is carried out with subject performing activities of daily living while monitored through wearable systems. A two-lead portable electrocardiograph was used during the experimental protocol. ECG Signals and derived parameters were analyzed to assess signal quality and its processing possibility with respect to standard silver-silver chloride electrodes as reference. The method was applied to a case study to demonstrate applicability and results with silver based textile electrodes.
\end{abstract}

Index Terms - Smart textile, wearable sensors, reliability assessment, textile electrodes.

\section{INTRODUCTION}

$\mathrm{T}$ he future of healthcare is based on the development of new technologies and systems for a more accurate, personalized and widespread diagnosis and treatment of pathologies.[1]. In the biomedical engineering field, the role of technological innovation is strategic and fundamental to improve the quality of care.

Among the most relevant innovations, Personal Health Systems (PHS) are a recent concept (late 1990s), that are completely changing the way in which healthcare is provided. They were introduced as further development of health services by exploiting the innovation in science and technology such as the biomedical, micro-and nanotechnologies, and the innovation in the Information and Communication Technologies (ICT). PHS have been designed to place the individual citizen/patient in the center of the

Manuscript received October 9, 2012. This work was partially by a PRIN 2009 Grant entitled: "TELEFETALCARE: Design of a wearable system for remote fetal monitoring during pregnancy".

G. Andreoni I. Witkowska, P. Perego, M. Fusca, and M. Mazzola are with the Politecnico di Milano, Design Department, Via Durando 38/A, 20158, Milano, Italy (phone: +39 23998881 fax: +39 2399 7280; e-mail: giuseppe.andreoni@polimi.it)..

A. Fanelli and M. G. Signorini are with the Politecnico di Milano, DEIB Department, Piazza Leonardo da Vinci 32, 20133, Milano, Italy (phone: +39 23999501 fax: +392399 9508; e-mail: andrea.fanelli@ mail.polimi.it) healthcare delivery process. They allow citizens/patients to have more responsibility in managing their own health and interacting, whenever is necessary, with care providers. In doing so, PHS aim to bring benefits to citizens and health authorities alike: first, by improving the quality of care for the individuals themselves; second, by containing the rising healthcare costs through proper and efficient use of technological capabilities [1].

Wearable Biomedical Systems (WBS) are a specific category of PHS: in particular, they can be defined as integrated systems on a wearable platform (in the sense of clothing or devices attachable to the human body) and can offer solutions for continuous monitoring by measuring noninvasive biomedical, biochemical and physical parameters. Continuous monitoring with early detection of anomalies has likely the potential to provide patients with an increased level of confidence, which in turn may improve the quality of life. In addition, ambulatory monitoring will allow patients to engage in practicing normal activities of daily life, rather than staying at home or close to specialized medical services. Thus WBS are an ideal platform for multi-parametric non-intrusive monitoring of health status, thus providing a remote primary and secondary prevention. In this way it is possible to obtain the early diagnosis and management of several diseases (in particular cardiovascular and / or respiratory, but also metabolic pathologies and physical rehabilitation), but also to support elderly and disabled people [2].

WBS are used to measure a variety of biological signals, including heart rate (ECG), electrical cerebral activity (EEG), respiration, blood gases saturation, body accelerations, and many others. For example, in case the necessity is to guarantee a general monitoring of physiological parameters, a good balance between system's complexity and user's compliance might be obtained by a wearable device monitoring 1 or 2 ECG leads, breathing parameters (rate but also in/expiration times and volumes), and global motion (through one1 3-axial accelerometer).

Up to now, several studies have been conducted to support new services to monitor physiological parameters of people at home during activities of daily living (ADL) $[3,4,5,6]$. Despite this wide literature of new methods and services, rarely the performance of sensors have been validated by using a standardize protocol. Even if validation has been conducted 
for motion sensors like MEMS, used to detect movements and in particular falls $[7,8,9,10,11]$, we couldn't find any validation study to quantify the performance of wearable sensors.

Nevertheless, textile electrodes to detect ECG signal have been often integrated in wearable systems to have a more complete description of the health status of patients $[1,3,4]$. For these sensors the only validation is generally limited to a qualitative observation of the ECG waveforms to assess a good similarity in terms of shape and temporal patterns with a clinical ECG signal obtained with clinical electrodes and devices.

Standard electrodes for ECG monitoring are a class I medical device and generally are adhesive silver/silver chloride $(\mathrm{Ag} / \mathrm{AgCl})$ electrodes because of its ease of manufacturability. $\mathrm{Ag} / \mathrm{AgCl}$ electrodes are non-polarized electrodes as they allow current to pass across the interface between the electrolyte and the electrode [12]. Non-polarized electrodes are better than polarized electrodes in terms of their rejection of motion artifacts and their response to defibrillation currents.

Instead textile electrodes are built of textile yarns with electrical properties: a) Metal yarns, i.e. yarns containing conductive fibers like stainless steel, copper or silver mixed with natural or synthetic fibers; b) Yarns containing electroconductive fibers like polymeric or carbon coated threads [13].

This paper presents the a structured protocol for textile electrode validation. We compared the performances of ECG textile electrodes with standard $\mathrm{Ag} / \mathrm{AgCl}$ electrodes not only by looking at the morphological shape of the waveforms of the recorded ECGs, but also by verifying that the diagnostic information is preserved. In order to test our protocol, we employed it on a case study and we assessed the reliability of the textile electrodes under analysis.

\section{Materials AND MethodS}

\section{A. Experimental protocol and data acquisition}

The validation protocol for textile electrodes consisted in 2 phases:

- a laboratory test for measuring the conductivity and the sensing performances of textile electrodes in a fixed setup using a ECG signal generator;

- a functional set of experiments in real conditions while the subjects performed a standard ADL using a compact and portable clinical electro-cardiograph (ECGraph).

The first step aimed at assessing the "absolute" sensing capability of the textile electrodes, i.e. without any dependencies on the presence of the subject. The experimental set up consisted in a ECG generator (MiniSim 1000, Netech Corporation, Farmingdale, NY, USA) and a clinical electrocardiograph (Phedra, SXT - Sistemi per Telemedicina, Lecco, Italy). The MiniSim 1000, Advanced MultiParameter Simulator is a powerful, comprehensive patient simulator in a compact case. It is designed to test the performance of patient monitoring instrumentation quickly and easily. The ECG generator provides full 12 lead ECG simulation with 14 user selectable rates from 30 to $350 \mathrm{BPM}$ and 14 user selectable amplitudes from 0.15 to $5 \mathrm{mV}$. Firstly, we recorded the signal by directly attaching the ECGgraph clips to the ECG generator (Figure 1) and by acquiring 10 ECG waveforms, generated using different amplitude and frequency settings; then we repeated the same measures by interfacing a pair of $1 \times 1.5 \mathrm{~cm}$ textile electrodes (Comftech s.r.l., Monza, Italy) built with polymeric yarns with a silver-based coating and by mounting the standard ECGraph snaps. To compare the two sets of signals we computed the maximum of the normalized crosscorrelation coefficient, the QRS correlation coefficient and the QRS amplitude ratio.

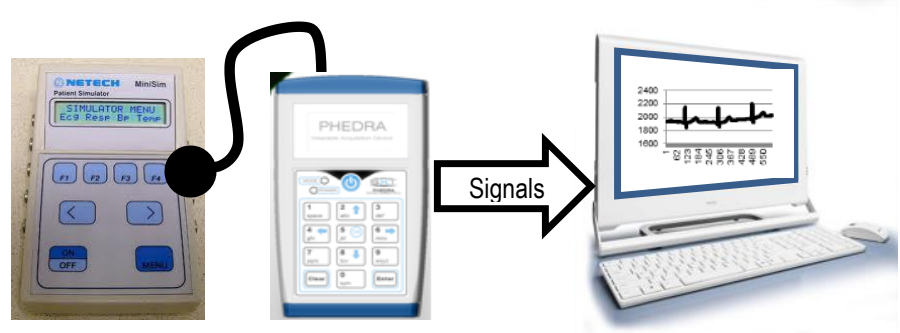

Fig. 1. The experimental setup for the validation of the electrodes using an ECG signal generator (on the left) whose output signals are recorder through a clinical ECGraph (in the center) and transmitted to a PC (on the right) for display and further processing.

The second phase consisted in an on-field test in which ECG signals were acquired from subjects performing standard ADL. The study was conducted on 10 healthy adults at the Politecnico di Milano, Milano, Italy. 7 men and 3 women participated in the research. The characteristics of the study group are shown in Table 1. Table 2 summarizes avg \pm std values for the male and female populations.

\begin{tabular}{|l|l|l|l|l|l|}
\hline Subject & Sex & $\begin{array}{l}\text { Age } \\
(\text { yrs })\end{array}$ & $\begin{array}{l}\text { Height } \\
(\mathbf{c m})\end{array}$ & $\begin{array}{l}\text { Body } \\
\text { Mass }(\text { Kg })\end{array}$ & BMI \\
\hline $\mathbf{1}$ & $\mathrm{M}$ & 31 & 180 & 80 & 24,69 \\
\hline $\mathbf{2}$ & $\mathrm{M}$ & 28 & 174 & 68 & 22,46 \\
\hline $\mathbf{3}$ & $\mathrm{M}$ & 32 & 180 & 84 & 25,93 \\
\hline $\mathbf{4}$ & $\mathrm{M}$ & 47 & 182 & 80 & 24,15 \\
\hline $\mathbf{5}$ & $\mathrm{M}$ & 28 & 178 & 78 & 24,62 \\
\hline $\mathbf{6}$ & $\mathrm{M}$ & 26 & 186 & 68 & 19,66 \\
\hline $\mathbf{7}$ & $\mathrm{M}$ & 42 & 186 & 103 & 29,77 \\
\hline $\mathbf{8}$ & $\mathrm{F}$ & 26 & 160 & 53 & 20,70 \\
\hline $\mathbf{9}$ & $\mathrm{F}$ & 30 & 167 & 53 & 19,00 \\
\hline $\mathbf{1 0}$ & $\mathrm{F}$ & 28 & 167 & 57 & 20,44 \\
\hline Average & ---- & 31.8 & 176 & 72.4 & 23.142 \\
\hline SD & ---- & 7.1 & 8.8 & 15.8 & 3.3 \\
\hline
\end{tabular}

Table 1 Average and standard deviation $( \pm$ SD) values of age, weight, body mass and BMI

During this second phase, the experimental setup consisted of a 5 electrodes configuration to simultaneously record the trunk 
lead (Figure 2) - which is the preferred one for wearable monitoring applications [14] - both with standard $\mathrm{Ag} / \mathrm{AgCl}$ and textile pairs of electrodes.

\begin{tabular}{|l|l|l|l|l|l|}
\hline $\begin{array}{l}\text { SEX } \\
(\mathbf{M} / \mathbf{F})\end{array}$ & & AGE & $\begin{array}{l}\text { HEIGH } \\
\text { T }(\mathbf{c m})\end{array}$ & $\begin{array}{l}\text { BODY } \\
\text { MASS } \\
(\mathbf{K g})\end{array}$ & BMI \\
\hline \multirow{2}{*}{$\mathbf{M}$} & AVG & 33.43 & 180.86 & 80.14 & 24.47 \\
\cline { 2 - 6 } & SD & 7.96 & 4.30 & 11.81 & 3.10 \\
\hline \multirow{2}{*}{$\mathbf{F}$} & AVG & 28.00 & 164.67 & 54.33 & 20.05 \\
\cline { 2 - 6 } & SD & 2.00 & 4.04 & 2.31 & 0.91 \\
\hline
\end{tabular}

Table 2 Average and standard deviation $( \pm \mathrm{SD})$ values of age, weight, body mass and BMI

The two pairs of electrodes were mounted as close as possible to each other in order to have the most similar signal as possible. No skin treatment was adopted both for men and women (neither skin preparation with cleaning solution nor shaving the electrode sites). In order to have the same electrode typology (non-polarized class) a small amount of gel for ECG recordings was placed on the textile electrodes before positioning them onto the body. This operation could be questionable for the comparison; in fact one of the main issues for textile electrode is whether to use gel (usually hydrogel pads could be placed in correspondence of the electrodes, as seen in some previous experiences) or leave dry electrodes. In our experience, a small sweat is produced at the skin-electrode interface, and this phenomenon is accelerated by activity, like stepping or stair climbing. Then the condition is nearly stable. Due to the short duration of laboratory test per subject (see next comma for details), we decided to anticipate this "stable" condition with a drop of gel onto the textile electrodes to have comparable results along the trial and among the trial as well.

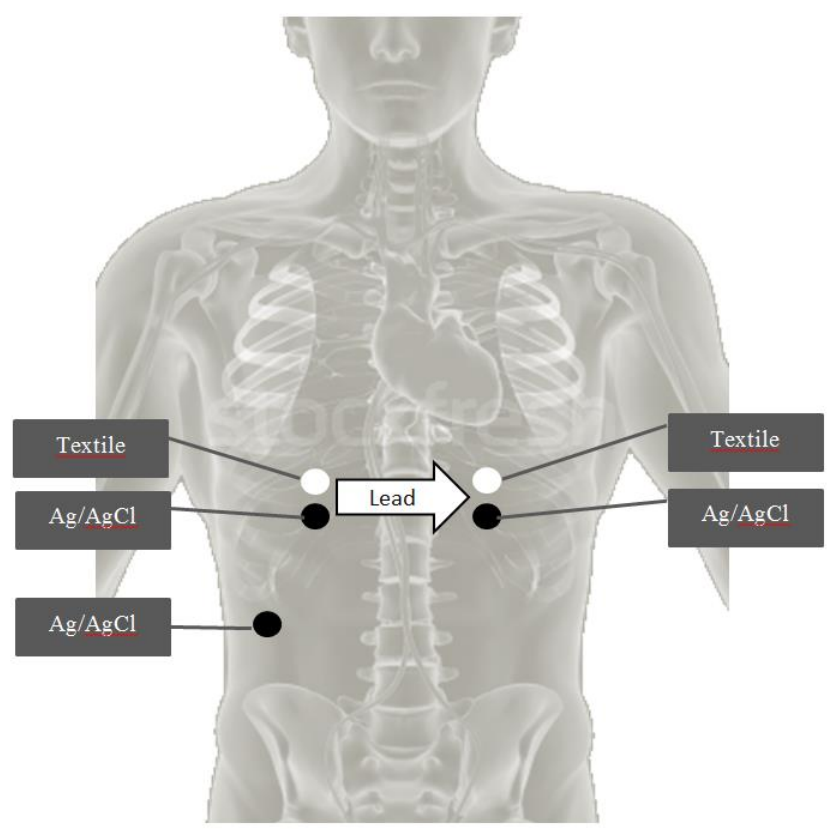

Fig. 2. The electrode configurations used in the experimental protocol.

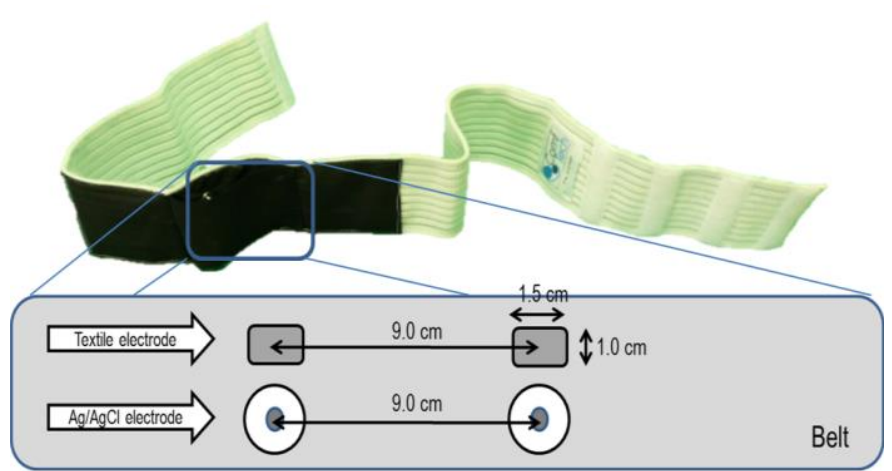

Fig. 3. The belt for supporting the 2 pairs of electrodes and their configuration in the experimental tests.

The clinical ECGraph (Phedra, SXT - Sistemi per Telemedicina, Lecco, Italy) was used to acquire ECG with 128 $\mathrm{Hz}$ sampling rate. The device was attached to the trunk of the subject through an elastic belt adjustable according to the dimension of the chest (figure 3). The ECGraph simultaneously records and sends data via Bluetooth to the PC, where the signals are displayed to have a real-time qualitative feedback of the signals quality.

Each trial consisted in a set of ADL epochs to be performed sequentially:

- Standing $30 \mathrm{sec}$

- Stand-to-sit (5 sec), stop, sit-to-stand (5 times)

- Standing $30 \mathrm{sec}$

- Stepping $30 \mathrm{sec}$

- Walking $30 \mathrm{sec}$

- Stairs (9 steps), up and down (2 times)

- Walking $30 \mathrm{sec}$

- Standing $30 \mathrm{sec}$

- Sitting $30 \mathrm{sec}$.

The duration could seem short but it was previously tested on two subjects that a longer recording during the performance of the same activities lead to constant results. For this reason we focused on 30 seconds recording of steady testing conditions, and we chose to verify repeatability.

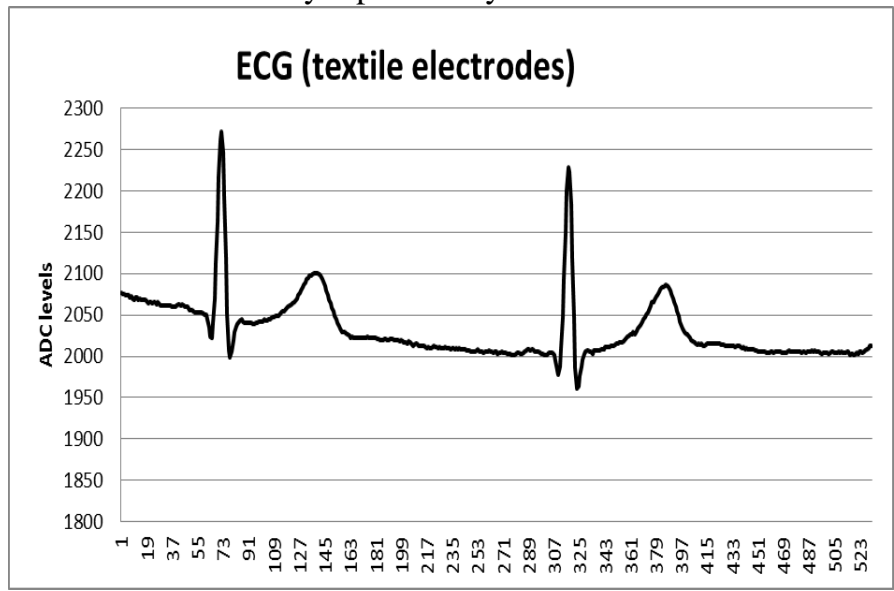

Fig. 4. An example of the ECG signal from textile electrodes in the experimental tests with subjects. 
Three repetitions of the sequence were carried out and recorded for each subject; each trial was followed by a 5 minutes sitting resting time, before starting a new one. In 3 subjects the positions of the electrodes were exchanged to verify absence of differences due to the positioning of the different kind of electrodes. For more clarity, in these subjects the textile electrodes were mounted upper than the standard electrodes with respect to the configuration shown in fig. 2 .

\section{B. Data Analysis}

The main purpose of the analysis was to compare the signals acquired using standard electrodes with the ones obtained using textile electrodes. The acquisition protocol was designed in order to acquire simultaneous data with the two different sensor technologies.

As first step, recordings were segmented according to the different protocol epochs (described above) by means of an automatic approach. This allowed a direct comparison of the signals obtained under the same experimental epoch. After that we computed a set of parameters in order to quantify similarity between pairs of corresponding recordings. The parameters were computed on the recordings acquired both with traditional and textile electrodes.

\section{QRS detection and evaluation}

We proceeded by quantifying their temporal and morphological characteristics. One of the most relevant diagnostic information provided by ECG is represented by heart rate variability, which is the sequence of the temporal inter-beat distance as a function of time. For a reliable and diagnostically significant ECG recording, it is extremely important to avoid distortions in ECG waveform that might cause erroneous interpretation of these physiological data.

The main fiducial point of the ECG waveform is the R peak of the QRS complex, so we proceeded by detecting QRS temporal position. In order to detect QRS, we employed an automatic algorithm previously developed by Fanelli et al. [15]. The approach is based on a cross-correlation template matching approach, which allows the identification of the precise temporal sample in which the $\mathrm{R}$ peak occurs. For more details about the implementation of the algorithm, please refer to [15].

Then, we computed:

- The errors in QRS detection, by considering as reference the QRS detection in recordings acquired with standard electrodes;

- The average absolute temporal distance (in sample) between the QRS detected in recordings acquired with textile electrodes and the QRS detected in recordings acquired with traditional ones. Every temporal distance over $100 \mathrm{~ms}$ is considered an error of detection.

- The average value of the ratio between the peak to peak amplitude of corresponding (synchronous) QRSs.

As a further preprocessing step, we removed the signal baseline thanks to a FIR high pass filter (transition band 0.05$1 \mathrm{~Hz}$ ). Baseline removal was a fundamental task to allow a correct comparison of signals in terms of morphological characteristics.

After this step, we computed the normalized cross correlation between each pair of corresponding ECG waveforms (one from textile and the other one from traditional electrodes). This task was accomplished in order to evaluate how much the textile electrodes might cause a change in ECG in terms of shape and temporal pattern. Indeed the capacitive coupling of the textile electrodes with the skin might cause an integration of the electronic currents, thus changing the shape of the signal. This undesired condition could be at the basis of wrong interpretation of ECG.

The cross - correlation was computed by using the following equation:

$$
\begin{aligned}
& \widehat{R_{\max }^{2}}=\max _{m} \hat{R}_{x y}(m) \\
& =\max _{m} \begin{cases}\sum_{\substack{n=0 \\
\hat{R} x y(-m)}}^{N-1} x_{n+m} y_{n}^{*} & m \geq 0\end{cases} \\
&
\end{aligned}
$$

where $x$ and $y$ are the two ECG waveform to be compared and $N$ is the number of samples in the signal. In our case, we employed a window 90 samples long centered in the detected QRS (corresponding to $0.7 \mathrm{~s}$, the average length of a heart beat in an adult). The normalization is accomplished so that the autocorrelations of the signals at zero lag are identically 1. Each protocol epoch is then described by a single crosscorrelation value, which is obtained as the average value of all beat cross-correlations.

Table 3 lists all the computed parameters, providing a short description for each of them.

\begin{tabular}{|l|l|}
\hline Parameter & Description \\
\hline $\begin{array}{l}\text { QRS detection } \\
\text { Error }\end{array}$ & $\begin{array}{l}\text { Number of Errors in QRS detection by } \\
\text { taking recordings with traditional } \\
\text { electrodes as reference. }\end{array}$ \\
\hline $\begin{array}{l}\text { QRS Amplitude } \\
\text { Ratio }\end{array}$ & $\begin{array}{l}\text { Average ratio of the amplitude of } \\
\text { corresponding (synchronous) QRS. }\end{array}$ \\
\hline $\begin{array}{l}\text { QRS detection } \\
\text { Delay }\end{array}$ & $\begin{array}{l}\text { Average temporal distance (in sample) } \\
\text { between corresponding QRS. If Delay > } \\
25 \text { samples, it is considered as a wrong } \\
\text { detection }\end{array}$ \\
\hline $\begin{array}{l}\text { Beats cross } \\
\text { correlation }\end{array}$ & $\begin{array}{l}\text { Average value of the normalized cross } \\
\text { correlation between pairs of } \\
\text { corresponding beats. }\end{array}$ \\
\hline
\end{tabular}

Table 3 The parameters computed in the comparative analysis between standard and textile electrodes.

\section{MATH}

Results are split in the 2 different set of tests.

a) Validation of the electrodes with the ECG generator. 
This test was carried out to verity the reliability of the sensors in providing a signal of an excellent quality, thus suitable for further processing. For this purpose with did not compute derived parameters but we simply verified the correspondence of the acquired signal to the generated one.

The 40 recordings ( 20 for each electrode configuration) were properly coupled and we computed:

- the average cross-correlation between corresponding signals;

- the average amplitude ratio:

$$
A R=\left.\frac{1}{N} \sum_{i=1}^{N} \frac{Q R S_{\text {text }}}{Q R S_{d c}}\right|_{i}
$$

where $Q R S_{\text {text }}$ is the peak to peak amplitude of the $i$ QRS of the signal acquired using textile electrodes, $Q R S_{s t}$ is the peak to peak amplitude of the corresponding QRS in the signal acquired with direct contact between ECG simulator and the clip of the ECGgraph, and $N$ is the total number of QRS in the recording. The average values of cross-correlation and amplitude ratio for each experimental condition are shown in the following table 4.a and 4.b.

\begin{tabular}{|c|c|c|c|c|c|}
\hline $\begin{array}{c}\text { Frequency } \\
\text { (b/min) }\end{array}$ & 45 & 60 & 90 & 120 & 150 \\
$\begin{array}{c}\text { Amplitude } \\
(\mathrm{mV})\end{array}$ & 0.998 & 0.999 & 0.999 & 0.999 & 0.999 \\
\hline 0.15 & 0.999 & 0.999 & 0.999 & 0.999 & 0.998 \\
\hline 0.30 & 0.999 & 0.999 & 0.999 & 0.998 & 0.999 \\
\hline 0.50 & 0.999 & 0.999 & 0.999 & 0.999 & 0.998 \\
\hline 1.00 & 0.999 & 0.999 \\
\hline
\end{tabular}

Table 4.a - Average signal cross correlation between recordings acquired with direct contact vs textile electrode in between ECG generator device and ECGraph.

\begin{tabular}{|c|c|c|c|c|c|}
\hline $\begin{array}{c}\text { Frequency } \\
\text { (b/min) } \\
\text { Amplitude } \\
(\mathrm{mV})\end{array}$ & 45 & 60 & 90 & 120 & 150 \\
\hline 0.15 & 1 & 1 & 1 & 1 & 1 \\
\hline 0.30 & 1 & 1 & 1 & 1 & 1 \\
\hline 0.50 & 1 & 1 & 1 & 1 & 1 \\
\hline 1.00 & 1 & 1 & 1 & 1 & 1 \\
\hline
\end{tabular}

Table 4.b - Average signal Amplitude ratio between recordings acquired with direct contact vs textile electrode in between ECG generator device and ECGraph.

b) Validation of textile $v$ s standard electrodes in real conditions of ADL monitoring.

The 30 recordings ( 3 for each subject) were processed by separating each protocol epoch and then the parameters were computed according to the previously described methods. The following tables $5 . \mathrm{a} / \mathrm{b}$ and $5 . \mathrm{a} / \mathrm{b}$ summarize the result of the test concerning monitoring of real ADL in 10 subjects.

If the same activities are pooled we have the following recapitulating table $6 . \mathrm{a} / \mathrm{b}$.

\begin{tabular}{|c|l|l|l|l|}
\hline $\begin{array}{l}\text { Textile vs } \\
\text { standard } \\
\text { electrodes } \\
\text { comparison } \\
\text { (Average) }\end{array}$ & $\begin{array}{l}\text { QRS } \\
\text { detection } \\
\text { Error } \\
\text { (No.) }\end{array}$ & $\begin{array}{l}\text { QRS } \\
\text { Amplitude } \\
\text { Ratio } \\
(1=\text { std } \\
\text { electr.) }\end{array}$ & $\begin{array}{l}\text { QRS } \\
\text { Cross } \\
\text { Correlatio } \\
\text { n } \mathrm{R}^{2} \text { ) }\end{array}$ & $\begin{array}{l}\text { QRS } \\
\text { detection } \\
\text { delay } \\
\text { (samples) }\end{array}$ \\
\hline standing & 1.634 & 0.930 & 0.926 & 0.642 \\
\hline stand-to-sit & 1.785 & 1.028 & 0.806 & 0.832 \\
\hline standing & 1.595 & 0.894 & 0.892 & 0.566 \\
\hline stepping & 2.026 & 1.021 & 0.756 & 0.661 \\
\hline walking & 2.063 & 1.116 & 0.737 & 0.797 \\
\hline stairs & 2.535 & 1.040 & 0.765 & 1.321 \\
\hline walking & 2.801 & 1.037 & 0.763 & 0.525 \\
\hline standing & 1.557 & 0.922 & 0.916 & 0.731 \\
\hline sitting & 0.525 & 0.931 & 0.930 & 0.563 \\
\hline AVERAGE & 1.836 & 0.991 & 0.832 & 0.738 \\
\hline SD & 0.652 & 0.074 & 0.082 & 0.243 \\
\hline
\end{tabular}

Table 5.a - Averages of Reliability Parameters computed in all the recordings and conditions, for all trials (and repetitions) and for all subjects.

\begin{tabular}{|l|l|l|l|l|}
\hline $\begin{array}{l}\text { Textile vs } \\
\text { standard } \\
\text { electrodes } \\
\text { comparison } \\
\text { (Standard } \\
\text { Deviation) }\end{array}$ & $\begin{array}{l}\text { QRS } \\
\text { detection } \\
\text { Error(No. } \\
\text { ) }\end{array}$ & $\begin{array}{l}\text { QRS } \\
\text { Amplitude } \\
\text { Ratio } \\
(1=\text { std } \\
\text { electrodes } \\
\text { standing }\end{array}$ & $\begin{array}{l}\text { Q.734 } \\
\text { QRS } \\
\text { Cross } \\
\text { Correlatio } \\
\left.\mathrm{n}^{2}{ }^{2}\right)\end{array}$ & $\begin{array}{l}\text { QRS } \\
\text { detection } \\
\text { delay } \\
\text { (samples) }\end{array}$ \\
\hline stand-to-sit & 2.532 & 0.284 & 0.073 & 0.496 \\
\hline standing & 4.309 & 0.238 & 0.135 & 0.788 \\
\hline stepping & 3.562 & 0.315 & 0.171 & 0.444 \\
\hline walking & 1.797 & 0.582 & 0.124 & 0.696 \\
\hline stairs & 4.154 & 0.543 & 0.137 & 2.291 \\
\hline walking & 5.133 & 0.342 & 0.098 & 0.378 \\
\hline standing & 3.278 & 0.292 & 0.069 & 0.530 \\
\hline sitting & 0.963 & 0.270 & 0.053 & 0.510 \\
\hline
\end{tabular}

Table 5.a - Standard deviations of Reliability Parameters computed in all the recordings and conditions, for all trials (and repetitions) and for all subjects.

\begin{tabular}{|c|c|c|c|c|}
\hline $\begin{array}{l}\text { Textile vs } \\
\text { standard } \\
\text { electrodes } \\
\text { comparison } \\
\text { (Average) }\end{array}$ & $\begin{array}{l}\text { QRS } \\
\text { detection } \\
\text { Error(No. } \\
\text { ) }\end{array}$ & $\begin{array}{l}\text { QRS } \\
\text { Amplitude } \\
\text { Ratio } \\
(1=\text { std } \\
\text { electrodes } \\
\text { ) }\end{array}$ & $\begin{array}{l}\text { QRS } \\
\text { Cross } \\
\text { Correlatio } \\
\mathrm{n}^{2} \text { ) }\end{array}$ & $\begin{array}{l}\text { QRS } \\
\text { detection } \\
\text { delay } \\
\text { (samples) }\end{array}$ \\
\hline Standing & 1.595 & 0.915 & 0.911 & 0.646 \\
\hline stand-to-sit & 1.785 & 1.028 & 0.806 & 0.832 \\
\hline Stepping & 2.026 & 1.021 & 0.756 & 0.661 \\
\hline Walking & 2.432 & 1.076 & 0.750 & 0.661 \\
\hline Stairs & 2.535 & 1.040 & 0.765 & 1.321 \\
\hline sitting & 0.525 & 0.931 & 0.930 & 0.563 \\
\hline $\begin{array}{c}\text { AVERAGE } \\
\text { (n=10) }\end{array}$ & 1.816 & 1.002 & 0.820 & 0.781 \\
\hline SD & 0.729 & 0.064 & 0.081 & 0.279 \\
\hline
\end{tabular}

Table 6.a - Averages of Reliability Parameters computed in all the recordings and conditions, for all trials (and repetitions) and for all subjects for pooled activities. 


\begin{tabular}{|c|c|c|c|c|}
\hline \begin{tabular}{|l|} 
Textile vs \\
standard \\
electrodes \\
comparison \\
(Standard \\
Deviation) \\
\end{tabular} & \begin{tabular}{|l} 
QRS \\
detection \\
Error(No.)
\end{tabular} & \begin{tabular}{|l|} 
QRS \\
Amplitude \\
Ratio \\
(1= std \\
electrodes \\
) \\
\end{tabular} & $\begin{array}{l}\text { QRS } \\
\text { Cross } \\
\text { Correlatio } \\
n\left(\mathrm{R}^{2}\right)\end{array}$ & $\begin{array}{l}\text { QRS } \\
\text { detection } \\
\text { delay } \\
\text { (samples) }\end{array}$ \\
\hline standing & 3.480 & 0.281 & 0.148 & 0.477 \\
\hline stand-to-sit & 2.532 & 0.457 & 0.135 & 0.788 \\
\hline stepping & 3.562 & 0.315 & 0.171 & 0.366 \\
\hline walking & 4.651 & 0.323 & 0.135 & 0.373 \\
\hline stairs & 4.154 & 0.543 & 0.137 & 2.291 \\
\hline sitting & 0.963 & 0.270 & 0.053 & 0.510 \\
\hline
\end{tabular}

Table 6.b - Standard Deviations of Reliability Parameters computed in all the recordings and conditions, for all trials (and repetitions) and for all subjects for pooled activities.

\section{DISCUSSION}

The suggested methodology represents a quick and simple approach which could generally applied to validate systems of acquisition. The availability of proper equipment (ECG signal generator and clinical 2-3 leads ECGraph) is required but the computational procedures are easy to be implemented in computational environments and programs like Matlab $\odot$, used in this approach. Moreover, results are easy to obtain and to interpret.

Even if the experimental activity is easy to be performed, it allows the precise quantification of the reliability of the monitoring system in the main tasks of ADL. In fact such a structured protocol could be applied not only to validate simple sensors such as the textile electrodes tested in the proposed example, but also complete systems, including, for instance, portable devices for a global analysis. Therefore this protocol represents a good starting but fundamental step to assess the applicability of these monitoring systems to everyday situations, preventing data loss or corruption by artifacts. With proper adaptation the protocol could be applied to other signals (e.g. EMG, respiration, etc.) or sensors (like MEMS for movements monitoring).

\section{A. Validation of the electrodes with the ECG generator.}

The experiment was performed to verify the correspondence in terms of amplitude and shape betweensignal acquired with textile electrodes and the generated one. Even after varying amplitude and frequency of the generated signal, no differences were encountered (average $R^{2}=0.9988 \pm 0.00041$ ), both in the pattern and in the amplitude of the measured signal The tiny differences could be attributed to measurement noise. Thus this demonstrates the theoretical reliability of the textile electrodes.

\section{B. Validation of the textile vs standard electrodes in real conditions of ADL monitoring.}

The second set of test aimed at demonstrating the equivalence between textile electrodes and standard Silver/SilverChloride ones in real conditions. To have a similar behavior of the electrodes a small amount of liquid conductive gel for ECG was used before textile electrodes positioning. A big difference is the presence of an adhesive layer for the Silver/SilverChloride electrodes with respect to the textile ones that are simply kept in touch with the body through the pressure exerted by an elastic belt to which they are knitted. For this reason a decrease in performance of textile electrodes is expected for the motion artifacts deriving from the not stable body-electrode contact. The performed experimental test could also assess how this effect is relevant and in what conditions (i.e. ADL typologies) this artifact could be significant in real monitoring situations.

Globally, the experiments showed that the acquired signals with the textile electrodes are almost identical to those acquired with the Silver/SilverChloride ones: the average amplitude ratio is equal to $0.992 \pm 0.074$ (less than $1 \%$ decrease in amplitude, not significant) and the average Cross Correlation coefficient is $0.832 \pm 0.082$. For this second parameter the best performances are shown while the subject is standing $\left(\mathrm{R}^{2}=0,911 \pm 0.148\right)$ while the most critical condition is encountered when the subject is walking $\left(\mathrm{R}^{2}=\right.$ $0,750 \pm 0.135)$. In both cases, the variability of these parameters is very small. After watching the video of the tests, we argued that this was due to the oscillations of the device that was placed just over the electrodes. Because of its not minimal weight and dimensions, even small movement might cause a slight detachment of the belt from the body, so decreasing the applied elastic adherence force. Furthermore the electromyographic contribution of the pectoralis muscles could be overlapped to the ECG signal. Despite this electrical signal is at higher frequencies, its amplitude is relevant and could affect both baseline and pattern of the ECG signal. This explains why, while standing and sitting, the standard electrodes gave a better signal quality. However, during the movement (stand to sit, stepping, walking, stairs ascending/descending using also the arms as natural) the best signal amplitude was obtained from textile electrodes that were highly conductive and wet.

However in any condition, the quality of the signals was more than good. To quantify signal quality, we computed the errors in QRS detection and the delay in samples between the time when the R-peak occurred in the signal recorded through the textile electrodes with respect to the time of the same event in the signal acquired with the Silver/SilverChloride ones. Globally the average number of errors in the detection of QRS was $1.836 \pm 0.652$ on a set of about 38 beats (about $30 \mathrm{sec}$ acquisition duration for each phase at about 75 beats-perminute average heart rate for standing/sitting/walking/sit-tostand activities) that corresponds to a global error of $2.448 \pm 0.869 \%$. The delay in the QRS detection was always less than 1 sample except for the stairs ascending and descending; in this case we had the worst results with an average delay of $1.321 \pm 2.291$ samples. This means that the signals were perfectly synchronized and no pattern modifications were present. Quiet movements or resting situations gave the best and excellent results, as expected. In fact we obtained that, after increasing the intensity of the movement, the error is bigger. For example during stepping, walking and stairs ascending/descending, the number of errors 
in QRS detection is respectively 2.026, 2.432, and 2.535 ; vice versa when the body is static (standing or sitting) this value decreases at less than 1 (the minimum value is 0.525 while the subject is sitting).

\section{CONCLUSION}

From the methodological point of view a detailed structured protocol for testing wearable sensors, and in particular textile electrodes, have been proposed and applied in monitoring ADL. This scenario represents the next future challenge to be achieved in developing new healthcare services and models. The method and the related experimental activity was easy to be implemented and carried out. Quantitative and effective parameters of reliability were computed to quantify the similarity and correspondence between standard and innovative monitoring solutions.

Also important suggestions were elicited by this work. In fact, the results obtained in this research work suggested that there was no significant difference between the two different typologies of textile and $\mathrm{Agl} / \mathrm{AgCl}$ electrodes. However specific attention should be taken into account while monitoring movements including arms motion (the EMG activity of the pectoralis muscles could affect the signal in amplitude and in high frequencies) and artifacts due to change in sensors adherence could be noticeable in the signal pattern.

This study was conducted on a single sample of silver-based textile sensors in a given but standard and common configuration (trunk lead and textile electrode with thin layer gel for electrical skin coupling): other materials and other configuration will be now tested for a wider and exhaustive validation of sensors typologies and materials.

\section{REFERENCES}

[1] Lymberis A., Gatzoulis L., "Wearable Health Systems: from smart technologies to real applications", in Conf Proc 2006 IEEE Eng. Med. Biol. Soc. , 2006 pp. 6789-6792.

[2] De Rossi D. and Lymberis A., "New generation of smart wearable health systems and applications", IEEE transactions on information technology in biomedicine, vol. 9, no. 3, 2005, pp. 293-294.

[3] Andreoni G., Bernabei M., Perego P., Barichello A., Piccini L., "Example of Clinical Applications of Wearable Monitoring System". International Journal of Computer Research. vol. 18, no. 3/4, 2011, pp. 323-339.

[4] Di Rienzo M., Meriggi P., Rizzo F., Castiglioni P., Lombardi C., Ferratini M., Parati G.,'Textile technology for the vital signs monitoring in telemedicine and extreme environments". IEEE transactions on information technology in biomedicine. vol. 14, no. 3, 2010, pp. 711717.

[5] Gilsoo Cho, Smart Clothing: Technology and Applications, CRC Press, 2009.

[6] Muschiato S., Romero M. , Perego P., Costa F., Andreoni G.," Designing Wearable and Environmental Systems for Elderly Monitoring at Home", in Advances in Social and Organizational Factors, Vink P., Ed., CRC Press, 2012, pp. 463-469, ch. 48.

[7] Giansanti D., Macellari V., Maccioni G.. New wearable system for the step counting: validation against a gold standard. Rapporti ISTISAN 07/43, 2007, pp. 1-20. Available: http://www.iss.it/binary/publ/cont/0743.1200568284.pdf

[8] Cutti A.G., Ferrari A., Garofalo P., Raggi M., Cappello A., Ferrari A., "'Outwalk': a protocol for clinical gait analysis based on inertial and magnetic sensors", Med Biol Eng Comput, vol. 48, 2010, pp.17-25.
[9] Ferrari A., Cutti A.G., Garofalo P., Raggi M., Heijboer M., Cappello A., Davalli A.," First in vivo assessment of "Outwalk": a novel protocol for clinical gait analysis based on inertial and magnetic sensors", Med Biol Eng Comput, vol. 48, 2010, pp. 1-15.

[10] Bourke A.K., vandeVen P., Gamble M., O'Connor R., Murphy K., Bogan E., McQuade E., Finucane P., O'Laighin G., Nelson J., "Evaluation of waist-mounted tri-axial accelerometer based falldetection algorithms during scripted and continuous unscripted activities", Journal of Biomechanics , vol. 43, no. 15, 2010, pp. 30513057.

[11] Bernabei M., Preatoni E., Mendez M., Piccini L., Porta M., Andreoni G., "A Novel Automatic Method for Monitoring Tourette Motor Tics Through a Wearable Device", Movement Disorders, vol. 25, no. 12, 2010, pp. 1967-1972.

[12] Webster J. G., Ed., "Medical Instrumentation Application and Design", John Wiley \& Sons, 2006.

[13] De Rossi D., Lymberis A., Eds, Wearable eHealth Sysyems for Personalised Health Management, Amsterdam: IOS Press, 2004.

[14] Andreoni G., "Sistemi di sensori indossabili per il monitoraggio: dalla Ricerca al Mercato", in Sistemi indossabili intelligenti per la Salute e la Protezione dell'Uomo, Bonfiglio A., Cerutti S., De Rossi D., Magenes G., Eds, Bologna: Patron, 2008, pp. 357-384, ch. 13.

[15] Fanelli A., Signorini M.G., Ferrario M., Perego P., Piccini L., Andreoni G., Magenes G., "Telefetalcare: a first prototype of a wearable fetal electrocardiograph", in Conf Proc 2011 IEEE Eng Med Biol Soc., 2011, pp. 1-4. 